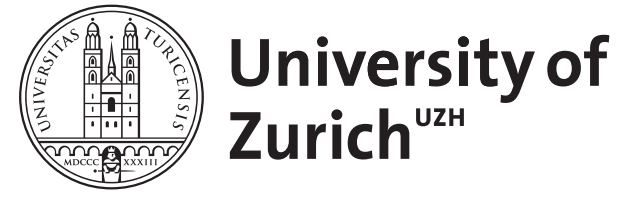

\title{
Drohende Frühgeburt
}

Beinder, E

\begin{abstract}
Frühgeburten haben mit über $70 \%$ einen erheblichen Anteil an der perinatalen Sterblichkeit sowie an der Morbidität von Neugeborenen. Die Ursachen der nichtiatrogenen Frühgeburtlichkeit sind mannigfaltig und umfassen aszendierende Infektionen, Mehrlingsschwangerschaften und Uterusfehlbildungen. Eine monokausale Therapie der drohenden Frühgeburtlichkeit kann es nicht geben. Es gibt auch kein Tokolytikum, das eindeutig die erste Wahl darstellt: Atosiban, Nifedipin, -Mimetika und Indometacin sind vermutlich äquieffektiv. Atosiban ist nebenwirkungsarm, aber sehr teuer. Nifedipin ist für die Behandlung in der Schwangerschaft nicht zugelassen. -Mimetika sind nebenwirkungsreich, es besteht allerdings die größte Erfahrung mit dieser Stoffgruppe für die Tokolyse. Nifedipin kann oral gegeben werden, während Fenoterol und Atosiban parenteral verabreicht werden müssen, um tokolytisch wirksame Konzentrationen zu erreichen. Intravenöses Magnesiumsulfat und Indometacin sollten in der klinischen Routine nur in besonderen Situationen für die Tokolyse eingesetzt werden. Andere Maßnahmen, wie die Verabreichung von Gestagenen, sind noch unzureichend erforscht oder - wie die Notfallcerclage - nur in ausgewählten Einzelfällen wirksam. Es gibt keine Evidenz dafür, dass additive Maßnahmen wie absolute Bettruhe, Hydratation und Sedation die drohende Frühgeburtlichkeit verbessern, sie sind deshalb im Regelfall nicht angezeigt
\end{abstract}

DOI: https://doi.org/10.1007/s00129-006-1813-6

Posted at the Zurich Open Repository and Archive, University of Zurich ZORA URL: https://doi.org/10.5167/uzh-156357

Journal Article

Published Version

Originally published at:

Beinder, E (2006). Drohende Frühgeburt. Der Gynäkologe, 39(4):299-310.

DOI: https://doi.org/10.1007/s00129-006-1813-6 
Gynäkologe 2006 39:299-310 DOI 10.1007/s00129-006-1813-6 Online publiziert: 24. März 2006

(c) Springer Medizin Verlag 2006

\section{Redaktion}

K. Vetter, Berlin

Die Frühgeburtlichkeit ist der Hauptgrund der perinatalen Mortalität und auch der schweren Morbidität. In den meisten westlichen europäischen Ländern beträgt die Frühgeburtlichkeit zwischen 7 und $8 \%$, deutlich höhere Raten werden aus den USA mit bis zu $12 \%$ berichtet. Es kann beobachtet werden, dass die Frühgeburtlichkeit weltweit nicht abnimmt, sondern eher ansteigt. - Anders die perinatale Mortalität, sie konnte insgesamt drastisch vermindert werden. Der bayerischen Perinatalerhebung zufolge nahm sie in Bayern im Zeitraum von 1979-2004 von 11 auf $4,8 \%$ ab. Dies ist allerdings vor allem den Fortschritten der Neonatologie zu verdanken und weniger geburtshilflichen Interventionen. Umfangreiche Bemühungen um die Problematik der nichtiatrogenen Frühgeburt haben zur Entwicklung einer Reihe von therapeutischen Ansätzen geführt, auf die im Folgenden eingegangen werden soll.

\section{Ätiologie}

Die Ursachen der Frühgeburt sind vielfältig (• Abb. 1). In etwa 30-40\% muss die Schwangerschaft aufgrund einer maternalen oder fetalen Gefährdung (z. B. Präeklampsie, fetale Wachstumsretardierung, Blutung bei Placenta praevia), also aus medizinischen Gründen beendet werden. Auf diese Ursachen soll hier nicht eingegangen werden.

Bei den übrigen $60-70 \%$ kommt es aufgrund eines vorzeitigen Blasensprungs, einer aufsteigenden Infektion, eines überdehnten Uterus (z. B. aufgrund von Mehrlingen oder Hydramnion) oder auch idiopathisch zu vorzeitigen Wehen. Die Entscheidung, einen Versuch zur Abwendung einer drohenden Frühgeburt zu unternehmen, muss immer individuell erfolgen. Dabei sollten die Schwangere bzw.

E. Beinder

Department Frauenheilkunde, Universitätsspital Zürich, Schweiz

\section{Drohende Frühgeburt}

die Eltern und ggf. Neonatologen einbezogen werden.

Im Einzelnen können folgende ätiologische/pathogenetische Kategorien unterschieden werden:

- Infektion: systemisch, aszendierend oder als Chorioamnionitis,

- fetale Ursachen wie Fehlbildungen mit Hydramnion und Mehrlinge,

- Uterusanomalien: Myome, Uterusfehlbildungen, Zervixinsuffizienz,

- idiopathisch,

- Plazentalokalisations- und -haftungsstörungen: Placenta praevia, Plazentarandblutung und Plazentalösung,

- Störungen der Plazentation und der Entwicklung der Plazenta mit konsekutiver Plazentainsuffizienz und

- mütterliche Ursachen (Präeklampsie, andere Erkrankungen in der Schwangerschaft).

Auf die ersten fünf Kategorien soll eingegangen werden. Klinisch äußern sich diese als Blasensprung, vorzeitige Wehentätigkeit, vaginale Blutung, Zervixeröffnung oder als mütterliche Beschwerden aufgrund des überdehnten Uterus.

\section{Früher vorzeitiger Blasensprung}

Ein früher vorzeitiger Blasensprung [vor der abgeschlossenen 37. Schwanger- schaftswoche (SSW), PPROM “preterm premature rupture of membranes"] tritt in etwa 5\% aller Schwangerschaften auf. Die sofortige Entbindung ist immer der prognostisch beste Weg für die Mutter, da hiermit die Gefahr infektiöser Komplikationen minimiert wird. Das Vorgehen bei PPROM muss sich deshalb immer an der Frage orientieren, ob die Risiken des konservativen Vorgehens [intrauterine Infektion (13-60\%), Plazentalösung (4-12\%), Nabelschnurvorfall (1-2\%, abhängig von der fetalen Lage)] die Risiken der Frühgeburtlichkeit aufwiegen. Derzeit bestehen folgende Empfehlungen:

- Nach der abgeschlossenen 34. SSW sollte die Geburt eingeleitet werden.

- Vor 32 (evtl. 34) SSW kann unter engmaschiger Kontrolle von Mutter und Kind abgewartet werden, sofern die stationäre Beobachtung in einem entsprechend ausgestatteten Perinatalzentrum erfolgt und eine manifeste intrauterine Infektion, ein fortgeschrittener Geburtsbefund, eine vaginale Blutung oder eine fetale Gefahr anhand von Kardiotokographie (CTG) und Ultraschall und eine genitale Herpesinfektion oder HIV-Erkrankung der Mutter ausgeschlossen sind.

Das Vorgehen besteht im Einzelnen aus: - Sicherung des Blasensprungs,
Tab. 1 Differenzialdiagnose der Blutung vor der Geburt

\begin{tabular}{|c|c|c|c|c|}
\hline Symptome & $\begin{array}{l}\text { Vorzeitige } \\
\text { Plazentalösung }\end{array}$ & Placenta praevia & $\begin{array}{l}\text { Insertio } \\
\text { velamentosa }\end{array}$ & Uterusruptur \\
\hline Schmerzen & \multirow[t]{3}{*}{++} & \multirow[t]{3}{*}{0} & \multirow[t]{3}{*}{0} & \multirow{3}{*}{$\begin{array}{l}++ \text { (manchma } \\
\text { stille Ruptur) }\end{array}$} \\
\hline $\begin{array}{l}\text { Hyperfrequente Kon- } \\
\text { traktionen }\end{array}$ & & & & \\
\hline Harter Uterus & & & & \\
\hline $\begin{array}{l}\text { Beginn mit Blasen- } \\
\text { sprung }\end{array}$ & 0 & 0 & ++ & 0 \\
\hline $\begin{array}{l}\text { Schocksymptomatik } \\
\text { der Mutter }\end{array}$ & +-++ & $0-++$ & 0 & $\begin{array}{l}++ \text { (Ausnah- } \\
\text { men möglich) }\end{array}$ \\
\hline
\end{tabular}




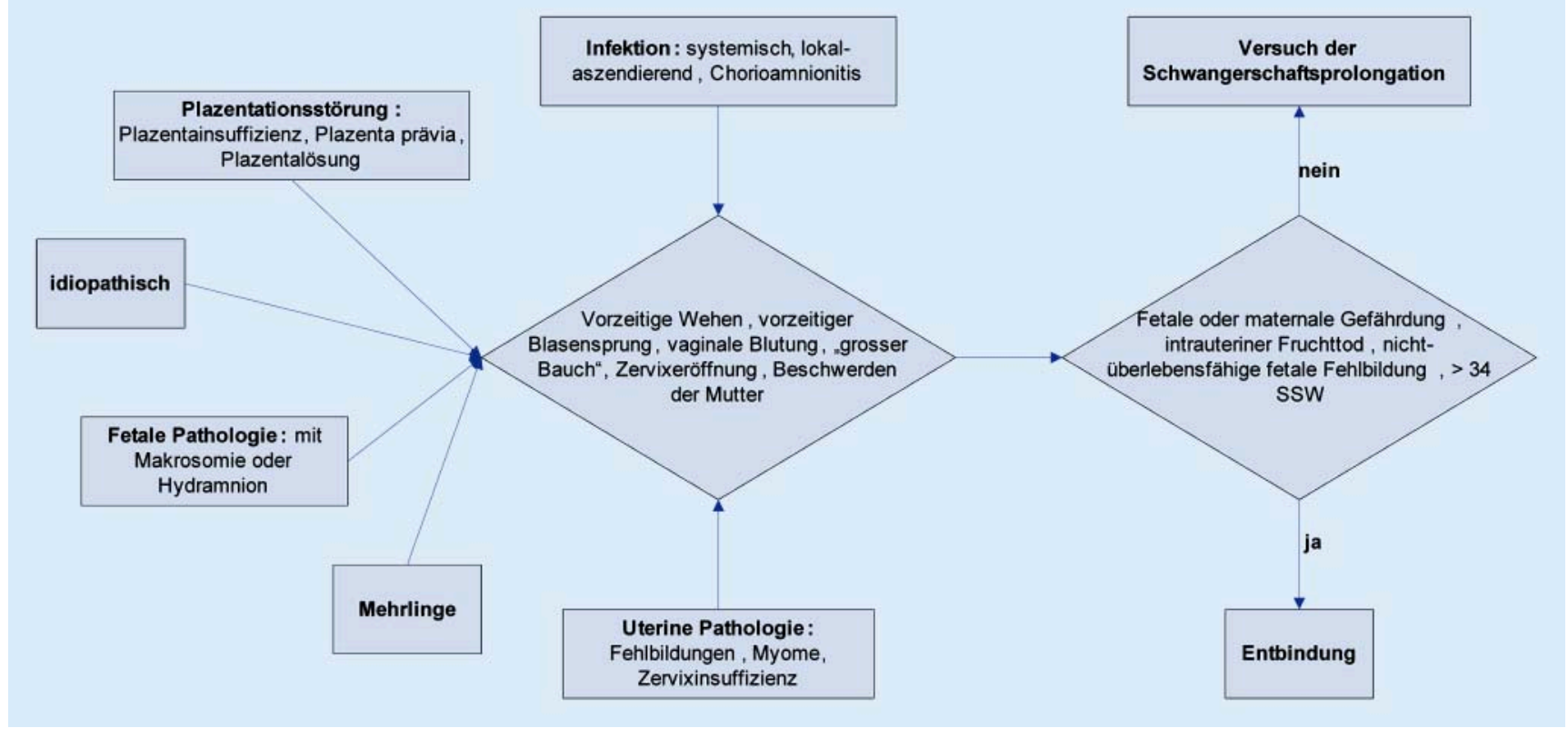

Abb. $1 \Delta$ Ursachen von vorzeitiger Wehentätigkeit und therapeutisches Vorgehen

- Ausschluss eines Amnioninfektionssyndromes (AIS):

Temperatur $>38^{\circ} \mathrm{C}$, mütterliche Tachykardie (>100-120 Spm), fetale Tachykardie (>16o Spm), druckschmerzhafter Uterus, zunehmende Wehentätigkeit, übel riechendes Fruchtwasser, Leukozytose (>20.00o/ $\mu \mathrm{l}$ ), CRP-Erhöhung (einmaliger Wert $>40 \mathrm{mg} / \mathrm{l}$, bzw. zwei Werte im Abstand von $24 \mathrm{~h}>20 \mathrm{mg} / \mathrm{l}$ ) sind charakteristische Zeichen,

- keine digital vaginale oder rektale Untersuchung, da dies die Latenzzeit bis zur Geburt nachweislich verkürzt,

- Zervix- und Vaginalabstrich zum Ausschluss einer pathologischen Keimbesiedlung, ggf. antibiotische Behandlung nach Antibiogramm,

- Eine prophylaktische Antibiose sollte bei PPROM immer erfolgen [13], ggf. zusätzlich nach Antibiogramm.

- Vor der 32. (evtl. 34.) SSW Durchführung der Lungenreifeinduktion mit Betamethason [24],

- Überwachung des Fetus mit CTG und Ultraschalluntersuchungen.

\section{Vaginale Blutungen}

Blutungen (• Tab. 1) treten in bis zu 3\% der Schwangerschaften auf und sind immer Anzeichen einer drohenden oder bereits bestehenden Gefahr für Mutter und
Kind. Sie bedürfen der sofortigen differenzialdiagnostischen Abklärung.

\section{Vorzeitige Plazentalösung (Abruptio placentae)}

Eine vorzeitige Plazentalösung tritt bei $0,4-1,3 \%$ aller Geburten auf und ist somit für bis zu $30 \%$ aller prä- und intrapartalen Blutungen verantwortlich.

Management. Bei schwerem Krankheitsbild der Mutter sind die schnelle Entbindung durch Sectio caesarea, die Schockprophylxe bzw. -bekämpfung sowie die Therapie der Blutung und der Gerinnungsstörung oberste Ziele.

Bei nur geringer randständiger Lösung der Plazenta (Plazentarandblutung oder Randsinusblutung) und noch erheblicher Frühgeburtlichkeit und fehlenden mütterlichen Symptomen kann unter stationärer Beobachtung abgewartet werden. Die Indikation zur Tokolyse muss allerdings sehr zurückhaltend gestellt werden und ist nur in Einzelfällen zu vertreten. Die perinatale Mortalität bei vorzeitiger Plazentalösung beträgt 20\%. Auch die Mutter ist in einzelnen schweren Fällen vital gefährdet. Die Wiederholungsrate in einer folgenden Schwangerschaft wird mit 5-15\% angenommen.

\section{Placenta-praevia-Blutung}

Die Häufigkeit beträgt 0,25-0,5\%. Die Diagnose einer Placenta praevia kann erst nach der 24. SSW zuverlässig gestellt werden, da vorher häufig Plazentaareale über dem inneren Muttermund beobachtet werden, die später nicht mehr nachweisbar sind (Hochwandern der Plazenta).

Management. Die Indikation zur Entbindung ist gegeben, wenn die Schwangerschaft älter ist als 34 SSW oder - unabhängig vom Schwangerschaftsalter - wenn die Blutung lebensbedrohlich für die Mutter ist oder wenn bei extrauterin lebensfähigem Feten Zeichen der drohenden fetalen Asphyxie im CTG auftreten.

Vor der 34. SSW kann ein konservatives Vorgehen angestrebt werden, wenn die Blutung spontan aufhört oder nur gering ist. Die Patientin wird dann stationär überwacht und behandelt. Dies umfasst: - Verabreichung einer niedrig dosierten Tokolyse, um den Uterus weitgehend ruhigzustellen. Es besteht keine Einigkeit in der Frage, ob die Tokolyse nach 48 Stunden wieder beendet werden soll oder ob eine Dauertokolyse durchgeführt werden soll. Evidenzbasierte Daten, die den Nutzen einer Dauertokolyse belegen könnten, fehlen

- Verabreichung von Anti-D-Immunglobulin bei allen Rhesus-negativen Frauen, um eine Sensibilisierung mit Rhesus-positivem fetalen Blut zu verhindern 
- Ständige Verfügbarkeit von Blutkonserven

- Tägliche CTG-Kontrolle, regelmäßige Beurteilung des kindlichen Wachstums und der Fruchtwassermenge, Dopplersonographie, u.a. mit dem Versuch, eine Placenta accreta oder increta auszuschließen.

\section{Uterusruptur}

Bei nicht voroperiertem Uterus sind Uterusrupturen extrem selten. Uterusrupturen werden nach Sectio mit isthmischem Querschnitt in 0,25\%, nach korporalem Längsschnitt (klassische Schnittführung) in 3-4\% unter der Geburt beobachtet. Die Rate an Uterusrupturen ist nach Enukleation transmuraler Myome mit Eröffnung des Uteruscavums (vor allem nach laparoskopischer Operation) und nach Operationen wegen uteriner Fehlbildungen (z. B. Strassmann-Operation) hoch. Die Uterusrupturgefahr bei Zustand nach Sectio mit isthmischem Querschnitt hängt auch davon ab, ob die Wehentätigkeit spontan eintritt (Risiko $0,52 \%)$ oder ob sie mit Oxytozin (0,77\%) oder mit Prostaglandinen (2,45\%) eingeleitet wird [20].

Management. Eine drohende Ruptur erfordert die sofortige Tokolyse und Durchführung einer Sectio caesarea. Bei eingetretener Ruptur oder Verdacht auf Uterusruptur ist eine sofortige Laparatomie notwendig, auch wenn der Fetus bereits verstorben ist, um die Blutung zu stillen und damit das Leben der Mutter zu retten. Bei kompletter Ruptur wird in der Regel die Hysterektomie durchgeführt, in besonderen Situationen (vor allem bei Narbenruptur) kann die alte Narbe ausgeschnitten und die Rupturstelle unter Erhalt des Uterus übernäht werden.

\section{Mehrlingsschwangerschaften}

Das am schwersten wiegende Risiko bei Mehrlingsschwangerschaften ist die Frühgeburtlichkeit. Das relative Risiko ist bei Zwillingen um den Faktor 5,4 gegenüber Einlingen erhöht. Bei höhergradigen Mehrlingen beträgt die Frühgeburtenrate annähernd $100 \%$ [3]. Interessanterweise zeigen verschiedene Untersuchungen, dass die Frühgeburtenrate bei Mehrlin-

Gynäkologe 2006 · 39:299-310～DOI 10.1007/s00129-006-1813-6

(c) Springer Medizin Verlag 2006

E. Beinder

Drohende Frühgeburt

\section{Zusammenfassung}

Frühgeburten haben mit über $70 \%$ einen erheblichen Anteil an der perinatalen Sterblichkeit sowie an der Morbidität von Neugeborenen. Die Ursachen der nichtiatrogenen Frühgeburtlichkeit sind mannigfaltig und umfassen aszendierende Infektionen, Mehrlingsschwangerschaften und Uterusfehlbildungen. Eine monokausale Therapie der drohenden Frühgeburtlichkeit kann es nicht geben. Es gibt auch kein Tokolytikum, das eindeutig die erste Wahl darstellt: Atosiban, Nifedipin, $\beta$-Mimetika und Indometacin sind vermutlich äquieffektiv. Atosiban ist nebenwirkungsarm, aber sehr teuer. Nifedipin ist für die Behandlung in der Schwangerschaft nicht zugelassen. $\beta$-Mimetika sind nebenwirkungsreich, es besteht allerdings die größte Erfahrung mit dieser Stoffgruppe für die Tokolyse. Nifedipin kann oral gegeben werden,

\section{Threatening premature birth}

\section{Abstract}

Preterm delivery contributes significantly to perinatal mortality and morbidity. Despite advances in obstetric care, the rate of such deliveries has increased over the past decade. The use of tocolysis, however, has only a minor impact on the prevention of preterm delivery because contractions do not necessarily induce delivery and prolongation of pregnancy is not desired in many cases for maternal or fetal reasons.

A clear first line tocolytic drug does not exist: nifedipine, atosiban, $\beta$-mimetics and indomethacin are equally effective. Atosiban has the lowest rate of maternal side effects but is expensive. According to the Cochrane Database, nifedipine is preferable to other tocolytics, however, it is not licensed for use in pregnancy. Most experience has been gained während Fenoterol und Atosiban parenteral verabreicht werden müssen, um tokolytisch wirksame Konzentrationen zu erreichen. Intravenöses Magnesiumsulfat und Indometacin sollten in der klinischen Routine nur in besonderen Situationen für die Tokolyse eingesetzt werden. Andere Maßnahmen, wie die Verabreichung von Gestagenen, sind noch unzureichend erforscht oder - wie die Notfallcerclage - nur in ausgewählten Einzelfällen wirksam. Es gibt keine Evidenz dafür, dass additive Maßnahmen wie absolute Bettruhe, Hydratation und Sedation die drohende Frühgeburtlichkeit verbessern, sie sind deshalb im Regelfall nicht angezeigt.

\section{Schlüsselwörter}

Vorzeitige Wehen · Tokolyse · Frühgeburt . Blasensprung $\cdot$ Mehrlinge

with $\beta$-mimetics which have several side-effects. Magnesium and indomethacin should not be used for routine tocolysis. The effectiveness for magnesium has not been demonstrated in randomized trials, and both of these substances have severe side-effects for the mother and/or fetus. There is no evidence in the literature that bed rest, hydration or the use of sedative drugs improve the rate of premature delivery.

\section{Keywords}

Premature contractions - Tocolysis · Preterm delivery · Rupture of membranes · Multiple pregnancy 


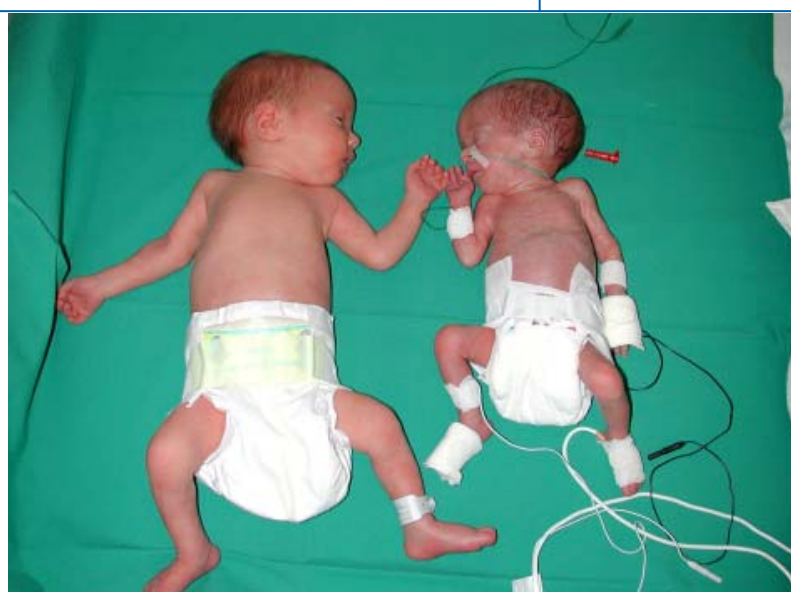

Abb. $2<$ Diskrepante dichoriale Gemini nach der Entbindung in der 33. SSW: Geburtsgewicht 2000 und $900 \mathrm{~g}$

gen im Lauf der Jahre angestiegen ist: in den USA beispielsweise stieg im Zeitraum 1995-1997 gegenüber dem Zeitraum 19811983 die Frühgeburtshäufigkeit von Zwillingen von 42 auf $54 \%$ an, während die allgemeine Frühgeburtlichkeit nur von 9,6 auf $11,1 \%$ anstieg [3]. Das durchschnittliche Entbindungsalter bei Zwillingen betrug nach einer Untersuchung von 2001 35,4, bei Drillingen 32, bei Vierlingen 29,6 und bei Fünflingen 29,1 SSW [21]. Die Frühgeburtenrate bei Mehrlingen ist gegenüber Einlingen stark erhöht, aber das perinatale Outcome ist gegenüber Einlingen desselben Gestationsalters ähnlich [2]. Zur Frühgeburtlichkeit bei Mehrlingen tragen folgende Faktoren bei:

- Erhöhte Wahrscheinlichkeit einer intrauterinen Wachstumsretardierung eines oder mehrerer Feten mit der Folge einer iatrogenen Frühgeburt (- Abb. 2).

- Auftreten eines fetofetalen Transfusionssyndroms bei monochorialen Gemini: Zwischen 10 und 20\% aller monochorialen Schwangerschaften entwickeln ein schweres fetofetales Transfusionssyndrom mit erhöhter perinataler Mortalität und/oder Frühgeburtlichkeit trotz therapeutischer Intervention (Amnionentlastung, Lasertherapie).

- Nabelschnurumschlingungen bei monoamnioten Zwillingen: Monoamniote Zwillinge sind zwar sehr selten (1 von 10.000 Schwangerschaften), aber die Gefahr von Nabelschnurumwicklungen beträgt etwa $70 \%$ und kann zum plötzlichen Tod beider Zwillinge oder zu pathologischen CTG-Befunden führen.
Die perinatale Mortalität monoamnioter Zwillinge wird mit 23\% angegeben [28]. Um die Gefahr eines unvorhersehbaren intrauterinen Fruchttodes zu vermindern, wird in den meisten Perinatalzentren die Sectio caesarea mit abgeschlossenen 32 bis 34 SSW durchgeführt. Es gibt aber auch Berichte, die dieses Vorgehen in Frage stellen, da Fruchttode bei monoamnioten Zwillingen vor 30 SSW häufig, danach aber selten sein sollen [4].

Bei drohender Frühgeburt und Mehrlingsschwangerschaft muss individuell abgewogen werden, ob eine Schwangerschaftsverlängerung für die Mutter subjektiv und objektiv zu tolerieren ist. Eine Tokolyse kann nach Ausschluss von Kontraindikationen angewendet werden, allerdings gibt es keine randomisiert kontrollierten Untersuchungen, die Tokolytika bei Mehrlingsschwangerschaften untersucht haben. Es ist zu beachten, dass die Gefahr von gravierenden mütterlichen Nebenwirkungen (z. B. Lungenödem) durch eine Tokolyse mit Sympathomimetika und wahrscheinlich auch Kalziumantagonisten bei gleichzeitiger Lungenreifeinduktion stark erhöht ist.

\section{Fetale Fehlbildungen}

Fetale Fehlbildungen gehen vor allem dann mit einer erhöhten Frühgeburtlichkeit einher, wenn sie zu einem Hydramnion führen. Dies ist dann der Fall, wenn eine Ösophagusatresie oder eine hohe Darmobstruktion bei Feten vorliegen, die das Schlucken bzw. die Resorption von Fruchtwasser unmöglich machen (- Abb. 3). Bei beginnenden Wehen ist in diesen Fällen die Fruchtwasserentlastung effektiver als die medikamentöse
Tokolyse. Selten kann auch ein fetaler Tumor (z. B. Riesen-Steißbeinteratome, fetaler Hydrops) zur Frühgeburt aufgrund einer Überdehnung des Uterus führen.

\section{Therapie}

\section{Gestagene}

Nach einer aktuellen Metaanalyse reduziert die prophylaktische Verabreichung von Progestagenen und 17a-Hydroxyprogesteroncaproat bei Schwangeren mit hohem Risiko die Frühgeburtlichkeit [31], während Untersuchungen zur Verwendung von Progestagenen bei symptomatischen Patientinnen bisher keinen Erfolg nachgewiesen haben [22].

\section{Notfallcerclage}

Als Notfallcerclage wird eine Cerclage bezeichnet, die im zweiten oder im frühen dritten Trimenon aufgrund einer bereits erfolgten Eröffnung der Zervix $>4 \mathrm{~cm}$ oder eines Fruchtblasenprolapses durchgeführt wird. Es liegen zu dieser Frage nur zwei kleinere prospektive Studien $(\mathrm{n}=\mathbf{2 2}$, $n=23$ ) vor: sie zeigen eine signifikante Verlängerung der Schwangerschaft um jeweils 4 Wochen gegenüber der Kontrollgruppe, die mit alleiniger Bettruhe behandelt wurden $[1,27]$. Dies weist darauf hin, dass durch die Notfallcerclage eine Verbesserung des perinatalen Outcomes in Einzelfällen erreicht werden kann. Aufgrund der unzureichenden Datenlage muss die Indikation zu einer Notfallcerclage allerdings immer individuell nach Ausschluss der Kontraindikationen gestellt werden.

\section{Weitere Maßnahmen}

Additive Maßnahmen wie absolute Bettruhe, Hydratation und Sedierung sind im Regelfall nicht angezeigt.

Antibiotika. Vorzeitige Wehentätigkeit, vor allem in frühen Schwangerschaftsstadien ist mit latenten Infektionen des oberen Genitaltraktes assoziiert. Es wurden deshalb zahlreiche Untersuchungen zur therapeutischen Verabreichung von Antibiotika bei vorzeitiger Wehentätigkeit und intakter Fruchtblase durchgeführt. Eine Cochrane-Metaanalyse von acht 


\section{Hier steht eine Anzeige.}

黛 Springer 


\begin{tabular}{|c|c|c|c|c|c|c|c|}
\hline & $\begin{array}{l}\text { Zugelassen } \\
\text { zur Tokolyse }\end{array}$ & $\begin{array}{l}\text { Wirksamkeit } \\
\text { gegenüber } \\
\text { Placebo }\end{array}$ & $\begin{array}{l}\text { Neben- } \\
\text { wirkungen }\end{array}$ & $\begin{array}{l}\text { Adminis- } \\
\text { tration }\end{array}$ & $\begin{array}{l}\text { Tages- } \\
\text { therapie- } \\
\text { kosten }\end{array}$ & $\begin{array}{l}\text { Wirksamkeit ge- } \\
\text { genüber anderem } \\
\text { Tokolytikum }\end{array}$ & $\begin{array}{l}\text { Schwangerschaftsverlän- } \\
\text { gerung von mindestens } \\
\text { 2-7 Tagen nachgewiesen }\end{array}$ \\
\hline$\beta$-Mimetika & $\begin{array}{l}\text { Fenoterol } \\
\text { (Partusisten }^{\circledR} \text { ) }\end{array}$ & Nachgewiesen & +++ & Intravenös & Mittel & Nachgewiesen & $\mathrm{Ja}$ \\
\hline Atosiban & $\begin{array}{l}\text { Atosiban } \\
\text { (Tractocile }^{\circledast} \text { ) }\end{array}$ & Nachgewiesen & + & Intravenös & Hoch & Nachgewiesen & $\mathrm{Ja}$ \\
\hline $\begin{array}{l}\text { Kalziumantago- } \\
\text { nisten }\end{array}$ & Nein & Nicht untersucht & +-++ & Oral & Gering & Nachgewiesen & $\begin{array}{l}\text { Ja (im indirekten Vergleich } \\
\text { mit anderen Tokolytika) }\end{array}$ \\
\hline Magnesium & $\mathrm{Ja}$ & $\begin{array}{l}\text { Nicht nachge- } \\
\text { wiesen }\end{array}$ & +++ & Intravenös & Mittel & $\begin{array}{l}\text { Nicht } \\
\text { nachgewiesen }\end{array}$ & Nein \\
\hline \multirow[t]{2}{*}{ Indometacin } & \multirow[t]{2}{*}{ Nein } & \multirow[t]{2}{*}{ Nachgewiesen } & + (Mutter) & \multirow[t]{2}{*}{ Oral } & \multirow[t]{2}{*}{ Gering } & \multirow[t]{2}{*}{ Nachgewiesen } & \multirow[t]{2}{*}{ Ja } \\
\hline & & & +++ (Fet) & & & & \\
\hline NO-Donatoren & Nein & $\begin{array}{l}\text { Datenlage noch } \\
\text { nicht ausrei- } \\
\text { chend }\end{array}$ & +-++ & $\begin{array}{l}\text { Transder- } \\
\text { mal }\end{array}$ & Gering & $\begin{array}{l}\text { Datenlage } \\
\text { uneinheitlich }\end{array}$ & $\begin{array}{l}\text { Datenlage nicht } \\
\text { ausreichend }\end{array}$ \\
\hline
\end{tabular}

randomisierten, placebokontrollierten klinischen Untersuchungen zeigte keinen Unterschied bezüglich Schwangerschaftsverlängerung, Frühgeburtlichkeit, neonatalem RDS und Sepsis [15]. Dies wurde auch in einer späteren multizentrischen Untersuchung bestätigt [14]. Die routinemäßige Anwendung von Antibiotika bei erhaltener Fruchtblase aus dem alleinigen Grund der Prävention der Frühgeburt ist somit nicht indiziert.

Immobilisierung. Es liegen keine prospektiv randomisierten Untersuchungen zur Bedeutung der Bettruhe in der Prävention oder Therapie vorzeitiger Wehentätigkeit bei Einlingsschwangerschaften vor. Für Zwillingsschwangerschaften konnte kein Benefit nachgewiesen werden. In der klinischen Erfahrung ist eine Reduktion körperlicher Aktivität bei erhöhtem Risiko für vorzeitige Wehentätigkeit oder bei eingetretener vorzeitiger Wehentätigkeit sinnvoll, es gibt aber keine Evidenz dafür, dass sie, vor allem wenn eine vollständige Immobilisierung eingehalten wird, die Frühgeburtlichkeit verringert.

Hydratation. Es gibt keine Evidenz, dass die intravenöse Hydratation die Frühgeburtlichkeit bei vorzeitiger Wehentätigkeit verringert. Bei Kombination der Hydratation mit der Lungenreifeinduktion des Feten und der Verabreichung kardiovaskulär wirksamer Tokolytika (v.a. $\beta$-Mimetika, Magnesiumsulfat und Kalziumantagonisten) besteht ein erhöhtes Risiko für das Auftreten eines maternalen Lungenödems.
Sedierung. Ebenso gibt es keine Evidenz dafür, dass die Sedierung der Mutter bei vorzeitiger Wehentätigkeit die Schwangerschaft verlängern kann oder einen anderen Benefit für Mutter und Kind aufweist.

\section{Medikamentöse Tokolyse}

Tokolytika kommen nur bei vorzeitiger Wehentätigkeit ohne Zeichen einer fetalen Beeinträchtigung oder einer maternalen Gefahr in Frage. Dies ist nur für einen geringen Teil der Fälle drohender Frühgeburt der Fall, weswegen die Tokolyse als alleinige Methode nur einen geringen Einfluss auf die Verminderung der Frühgeburtlichkeit haben kann. Durch Tokolytika kommt es - im Vergleich zu Placebo - zu einer signifikanten Verlängerung der Schwangerschaft um 2-7 Tage, ohne dass dies nach der vorliegenden Datenlage einen Einfluss auf die perinatale Mortalität hat [9].

Eine Reihe verschiedener Medikamente werden derzeit zur Tokolyse eingesetzt:

- $\beta$-Mimetika (Partusisten ${ }^{\circledast}$ ),

- Oxytozinrezeptor-Antagonisten

(Tractocile $^{\circledast}$ ),

- Kalziumantagonisten,

- Magnesium,

- Prostaglandinsynthasehemmer und

- NO-Donatoren.

Diese Substanzen unterscheiden sich in Wirksamkeit, Applikationsform, Nebenwirkungsprofil und Kosten (• Tab. 2, - Tab. 3)

\section{Indikationen}

Die frühgeburtsbedingte kindliche Morbidität und Mortalität ist bereits nach 34+o SSW gering. Eine medikamentöse Tokolyse ist aufgrund der Nebenwirkungen für die Mutter und des ungünstigen Nutzen/Risiko-Verhältnisses für den Feten nach 34+o SSW in der Regel nicht mehr indiziert. Individuelle Ausnahmen (z. B. Verlegung in ein perinatologisches Zentrum bei zusätzlichen fetalen Problemen) können bestehen.

Der Haupteffekt der Tokolyse besteht in einer Verlängerung der Schwangerschaft um 2-7 Tage gegenüber Placebo. Somit ist die Einleitung einer medikamentösen Tokolyse vor 22-24+o SSW in der Regel ebenfalls nicht indiziert. Auch hier gibt es individuelle Ausnahmen (z. B. perioperativ bei Cerclage; $\bullet$ Abb. 4).

Vorzeitige Wehen ohne Beeinflussung der Zervix werden häufig zu oft und zu lange medikamentös behandelt; dabei ist bekannt, dass 50-80\% aller Fälle mit vorzeitigen Wehen nicht zur Frühgeburt führen und daher auch nicht einer medikamentösen Wehenhemmung bedürfen [12]. Einer Studie zufolge werden zwischen 50 und 70\% der mit Placebo behandelten Schwangeren mit der klinischen Diagnose vorzeitige Wehentätigkeit, in Terminnähe entbunden [11].

\section{— Die zzt. besten prädiktiven}

Marker für eine echte Progredienz

vorzeitiger Wehen sind die

Zervixverkürzung und ein positiver

Fibronektintest im Vaginalsekret. 


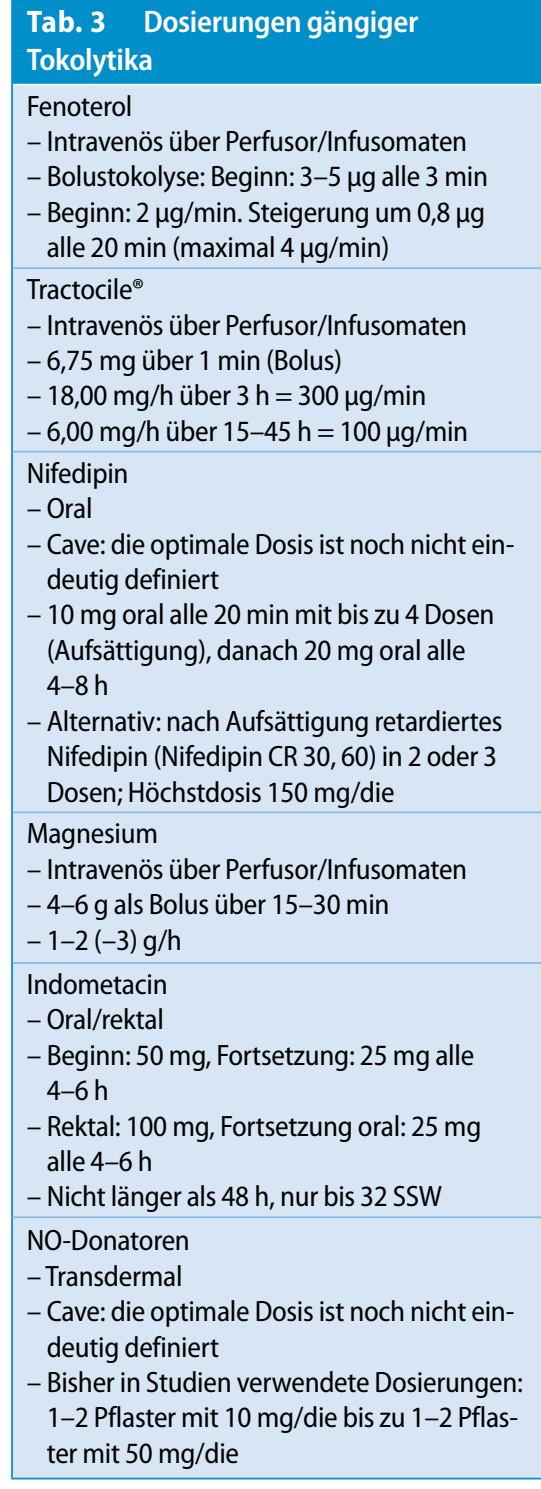

Eine medikamentöse Tokolyse sollte deshalb in der Regel nur erfolgen, wenn

- eine spontane vorzeitige Wehentätigkeit (schmerzhafte, palpable, länger als 30 s dauernde Kontraktionen, die häufiger als 3 pro 30 min sind) und eine funktionelle Zervixverkürzung zusammentreffen (• Abb.5),

- und/oder eine Muttermundserweiterung nachweisbar ist,

- und/oder der Fibronektintest im Vaginalsekret positiv ist.

Im Einzelfall oder in Hochrisikosituationen (z. B. höhergradige Mehrlinge, Blutung bei Placenta praevia) muss von diesen Kriterien abgewichen werden und nach individueller klinischer Entscheidung bereits bei Nachweis von regelmä-
Bigen Kontraktionen eine Tokolyse eingeleitet werden.

\section{Kontraindikationen}

Wehentätigkeit muss in erster Linie als maternaler „rescue“ bei drohender Gefahr für Mutter und Kind angesehen werden. Dementsprechend sind folgende Kontraindikationen vor einer medikamentösen Tokolyse auszuschließen:

- intrauterine Infektion,

- nicht mit dem Leben zu vereinbarende Fehlbildung des Fetus oder intrauteriner Fruchttod,

- mütterliche Indikation zur Schwangerschaftsbeendigung,

- kindliche Indikation zur Schwangerschaftsbeendigung,

- Schwangerschaftsdauer $>34$ Wochen.

\section{Wirkungen einzelner Tokolytika}

Nach dem derzeitigen Wissensstand sind Atosiban, $\beta$-Mimetika, Nifedipin und Indometacin äquieffektiv in der Hemmung vorzeitiger Wehen.

$\boldsymbol{\beta}$-Mimetika. $\beta$-Mimetika sind die am häufigsten verwendeten Medikamente zur Tokolyse. Sie binden an zellmembranständige $\beta$-adrenerge Rezeptoren. Eine Metaanalyse von King et al. 1998 [18] wies zwar die Effizienz von $\beta$-Mimetika hinsichtlich einer Prolongation der Schwangerschaft um 24-48 Stunden nach, nicht aber einen signifikanten Effekt auf die perinatale Mortalität

Atosiban. Atosiban ist ein kompetitiver, nichtselektiver Antagonist für Oxytocin und den Arginin-Vasopressin-Rezeptor, der oxytocininduzierte Kontraktionen unterdrückt. Romero et al. [20] zeigten in einer doppelt verblindeten, randomisierten Studie, dass Atosiban gegenüber Placebo bei vorzeitiger Wehentätigkeit signifikant effektiver ist in der Verlängerung der Schwangerschaft um 24 und 48 Stunden und um 7 Tage, eine Veränderung des perinatalen Outcomes war aber nicht nachweisbar. Moutquin et al. 2000 [23] wiesen in einer doppelt verblindeten randomisierten Studie nach, dass Atosiban und das $\beta$ Mimetikum Ritodrin äquieffektiv sind. Äquieffektivität von Atosiban und verschiedenen $\beta$-Mimetika wurde auch in einer internationalen Studie aufgezeigt [33].
Kalziumantagonisten. Kalziumantagonisten blockieren direkt den Influx von Kalziumionen durch die Zellmembran. In der Cochrane-Database wurden 12 randomisiert kontrollierte Studien mit 1029 Frauen zur Beurteilung der Wirkung zugrunde gelegt [17]. Kalziumantagonisten wurden dabei vor allem im Vergleich zu $\beta$-Mimetika als günstig eingestuft.

Indometacin. Prostaglandinsynthesehemmer wie Indometacin inhibieren konstitutive und induzierbare COX(Zyklooxygenase)-Enzyme, die für die Synthese von Prostaglandinen verantwortlich sind. Drei Untersuchungen verglichen Indometacin mit $\beta$-Agonisten: Bei kleinen Untersuchungszahlen (insgesamt 209 eingeschlossene Patientinnen) wurden keine Unterschied in der tokolytischen Effizienz festgestellt [18].

Magnesium. Magnesium wirkt intrazellulär als Kalziumantagonist. Dies führt zur Blockade von kalziumabhängiger Phosphorylierung der Myosinkinase und resultiert in einer myometranen Relaxation. Interessanterweise gibt es keine gute wissenschaftliche Untersuchung, die eine Wirksamkeit von Magnesium nachweist. Die einzige randomisierte, kontrollierte Untersuchung (Magnesiumsulfat vs. physiologische NaCl-Lösung) erbrachte keinen Wirksamkeitsnachweis [5], auch in einer Cochrane-Review wird es als ineffektiv für die Verzögerung der Geburt oder die Verhinderung der vorzeitigen Geburt beschrieben [7].

Nitroglyzerin. Eine neuere und in $\mathrm{Zu}$ kunft möglicherweise interessante Subs$\operatorname{tanz}$ ist Nitroglycerin. In der CochraneAnalyse von 2003, die allerdings nur 5 der 10 bisher publizierten Studien einbezieht, wird trotz eines günstigeren $\mathrm{Ne}$ benwirkungsprofils der Einsatz von Nitroglycerin-Pflastern aufgrund einer noch unzureichenden Datenlage nicht zur Routineanwendung empfohlen [8].

\section{Nebenwirkungen}

Das Nebenwirkungsprofil der einzelnen Tokolytika ist sehr unterschiedlich (- Tab. 2). 


\section{Zum Thema}
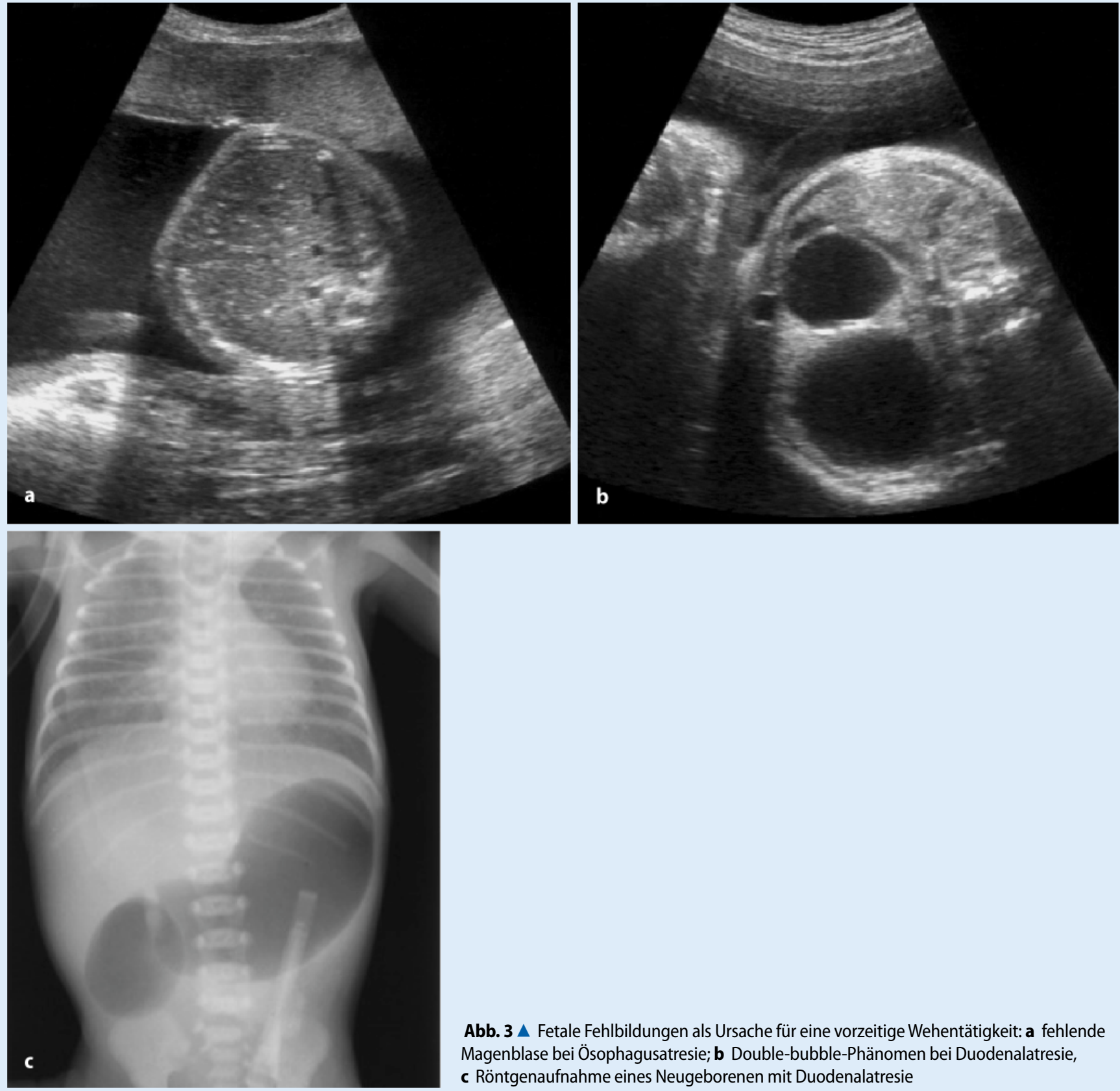

Abb. 3 Fetale Fehlbildungen als Ursache für eine vorzeitige Wehentätigkeit: a fehlende Magenblase bei Ösophagusatresie; b Double-bubble-Phänomen bei Duodenalatresie, c Röntgenaufnahme eines Neugeborenen mit Duodenalatresie

$\boldsymbol{\beta}$-Mimetika. Häufige Nebenwirkungen für die Mutter umfassen Palpitationen (48\%), Tremor (39\%), Übelkeit (20\%), Kopfschmerz (23\%) und Thoraxschmerzen (10\%). Schwere maternale Nebenwirkungen (v.a. Herzrhythmusstörungen und Lungenödem), die zu mütterlichen Todesfällen führten, sind beschrieben, wobei das Lungenödem in etwa 1:425 Fällen (nach manchen Berichten auch häufiger) auftritt [9]. Prädisponierende Faktoren sind Hydratation, gleichzeitige Präeklampsie oder Lungenreifeinduktion, Bluttransfusion, Mehrlingsschwangerschaften und mater- nale Herzerkrankungen. Die Bolustokolyse mit Fenoterol ist eine nebenwirkungsärmere Alternative zur kontinuierlichen Infusion von Fenoterol [32].

Eine maternale Herzerkrankung, Hypertonie, Hyperthyreose und eine schwere Anämie sind Kontraindikationen für die Verabreichung von $\beta$-Mimetika. Bei Diabetes mellitus der Mutter besteht eine relative Kontraindikation.

Atosiban. Atosiban weist das günstigste Nebenwirkungsprofil aller Tokolytika auf. Nebenwirkungen für die Mutter umfassen
Palpitationen (2\%), Tachykardie (6\%), Hypotonie (3\%), Übelkeit (12\%), Erbrechen (7\%) und Kopfschmerzen (10\%; [33]). Schwere Nebenwirkungen wurden unter alleiniger Therapie mit Atosiban bisher nicht beschrieben. Fetale Nebenwirkungen sind nicht bekannt. Das kindliche Follow-up aus zwei Studien, die Atosiban mit Placebo verglichen, zeigte im Alter von 24 Monaten keine signifikanten Unterschiede hinsichtlich mentalem, neurologischen und körperlichen Entwicklungsstatus. Als substanzspezifische Kon- 
Hier steht eine Anzeige.

至 Springer 


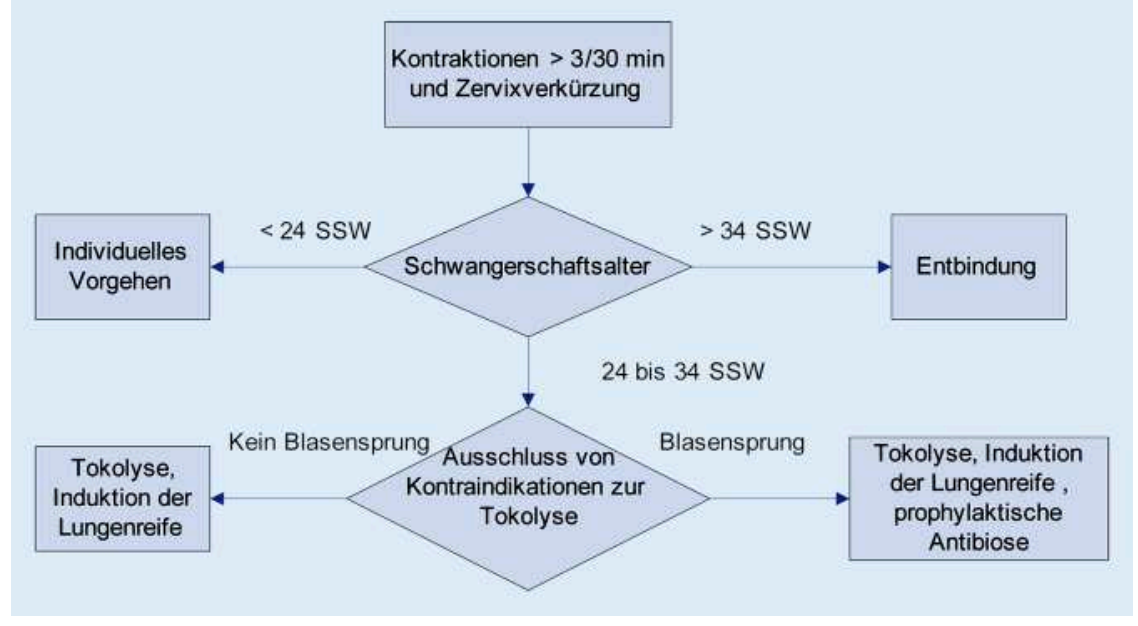

Abb. 4 \& Indikationsstellung zur Tokolyse

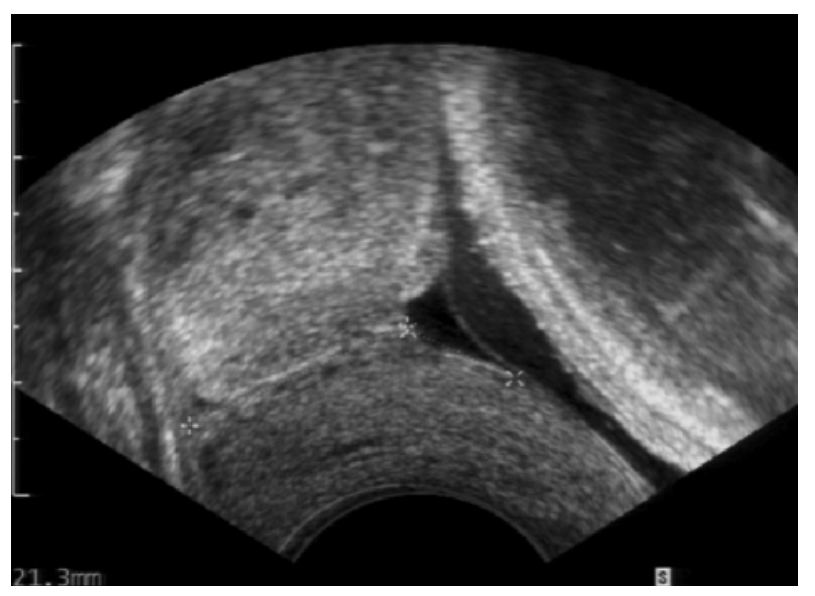

Abb. $5<$ Verkürzung der Zervix auf $21 \mathrm{~mm}$

traindikation wird lediglich die bekannte Überempfindlichkeit eingestuft.

Kalziumantagonisten. Auch Kalziumantagonisten weisen in der Regel wenige Nebenwirkungen auf. Vor allem in Kombination mit anderen kreislaufwirksamen Tokolytika wurde aber in Einzelfällen auch von schweren maternalen und/oder fetalen Nebenwirkungen berichtet.

Substanzspezifische Kontraindikationen sind die Hypersensitivität auf dieses Medikament, linksventrikuläre Dysfunktion, kongestive Herzfehler und Hypotonie der Mutter. Bei Einleitung einer Tokolyse mit Nifedipin sind engmaschigen Kontrollen von Mutter und Feten erforderlich.

Magnesium. Magnesiumsulfat in hoher Dosierung hat Effekte an der neuromuskulären Endplatte, woraus eine Schwäche und Abgeschlagenheit der Patientin resultiert. Schwere Nebenwirkungen von Atemdepression bis zu mütterlichen Todesfällen bei hohen Serumspiegeln von den Wirkstoff, peptische Magen-DarmUlzera, medikamenteninduziertes Asthma, hämatologische, renale oder hepatische Dysfunktion.

\section{Zulassung der Tokolytika}

Atosiban wurde in Europa von der EMEA (European Agency for the Evaluation of Medical Products) als Tokolytikum zugelassen. Die Zulassung in Kanada und den USA wurde von der FDA (Food and Drug Administration) abgelehnt. Fenoterol ist nur in Deutschland zur Tokolyse zugelassen.

Für Nifedipin, NO-Donatoren und Indometacin wurde die Zulassung als Tokolytikum weltweit von den Herstellern nie beantragt. Diese Medikamente sind somit für die Tokolyse nicht zugelassen. Interessant ist, dass in der WHO-Liste der „essential medicines" als einziges Antioxytocicum der Wirkstoff Nifedipin aufgeführt ist.

Nicht zugelassene Tokolytika sollten nur nach individueller Abwägung und ausführlicher Information der Patientin und Einholen des Einverständnisses für die Anwendung außerhalb der Zulassung angewendet werden.

\section{Dauertokolyse}

Auch nach initialem Ansprechen auf die Tokolysetherapie haben viele Frauen weiterhin ein hohes Risiko für vorzeitige Wehentätigkeit. Für die Erhaltungstherapie mit Atosiban gibt es eine placebokontrollierte Untersuchung: bei der kontinuierlichen s.c.-Verabreichung dauerte es in der Verumgruppe 33 Tage, in der Placebogruppe 27 Tage bis zum Wiederauftreten von Wehen [34]. Für alle anderen Tokolytika gibt es keine Evidenz für den Nutzen einer Dauertokolyse.

Aufgrund dieser beschränkten Studienlage besteht derzeit keine Evidenz dafür, dass eine Dauertokolyse in der klinischen Routine effektiver ist als die kurzfristige Tokolyse über 48 Stunden. Eine Dauertokolyse sollte also nur in ausgewählten Einzelfällen (z. B. symptomatische Placenta praevia und frühes Schwangerschaftsalter) erfolgen.

\section{Orale Tokolyse}

Es existieren mehrere Untersuchungen, die die fehlende tokolytische Wirkung von 
oral verabreichtem Magnesium nachweisen konnten [29]. Für oral verabreichte $\beta$ Mimetika wurden in randomisierten Untersuchungen widersprüchliche Ergebnisse gewonnen: Während eine prospektiv randomisierte, doppelt verblindete Untersuchung einen günstigen Effekt von oralem Ritodrin in einer Slow-release-Form nachwies [10], erbrachte die Mehrzahl der Untersuchungen keinen Vorteil [19]. Aus diesem Grund sollte heute eine orale Tokolyse mit Magnesium oder $\beta$-Mimetika nicht mehr erfolgen.

Nur für Nifedipin und Indometacin ist eine Wirksamkeit bei oraler Einnahme nachgewiesen, beide sind allerdings in Deutschland zur Tokolyse nicht zugelassen.

\section{Kombination von Tokolytika}

Auch unter Beachtung der substanzspezifischen Kontraindikationen sind bei alleiniger Anwendung von $\beta$-Mimetika, Nifedipin und Magnesiumsulfat in hoher Dosierung schwere mütterliche Nebenwirkungen beschrieben worden. Nifedipin kann schwere Komplikationen verursachen, wenn es in Kombination oder nach $\beta$-Mimetika und/oder hochdosiertem Magnesiumsulfat verabreicht wird $[26,35]$. Pharmakokinetische wie pharmakodynamische Daten in der Schwangerschaft sind für die meisten zur Tokolyse verwendeten Substanzen unbekannt.

Die Kombination von Tokolytika ist ungenügend untersucht, Nebenwirkungen nach dem derzeitigen Wissen unabsehbar. Sie ist deshalb in der klinischen Routine nicht angezeigt, sondern speziellen Situationen (z. B. offene Fetalchirurgie) vorbehalten.

\section{Additive Maßnahmen}

Entsprechend den Indikationen zur Verlegung in ein perinatologisches Zentrum gemäß der Leitlinie „Antepartaler Transport von Risikoschwangeren“ (Nr. 024/oo1) der Gesellschaft für Neonatologie und Pädiatrische Intensivmedizin sollte der Zeitgewinn bis zur Entbindung zur Verlegung der Schwangeren in ein Perinatalzentrum genutzt werden.

In dieser Zeit sollte ein einzelner Zyklus Betamethason (2-mal $12 \mathrm{mg}$ i.m. in Abstand von $24 \mathrm{~h}$ ) verabreicht werden [6].

\section{Fazit für die Praxis}

\section{Die Frühgeburtenrate steigt weltweit} an. Dennoch hat die perinatale Mortalität aufgrund der Fortschritte in der Neonatologie drastisch abgenommen. Aufgrund der multifaktoriellen Ursachen der Frühgeburtlichkeit ist eine monokausale Therapie nicht vorstellbar. Die medikamentöse Tokolyse kann die nichtiatrogene Frühgeburtlichkeit um 2-7 Tage gegenüber Placebo hinauszögern. Die Kontraindikationen der Tokolyse, allen voran das Amnioninfektionssyndrom, müssen beachtet werden. Atosiban ist nebenwirkungsarm, aber auch sehr teuer. Nifedipin ist für die Behandlung in der Schwangerschaft nicht zugelassen. $\beta$-Mimetika sind nebenwirkungsreich, es besteht allerdings die größte Erfahrung mit dieser Stoffgruppe zur Tokolyse. Weitere Maßnahmen zur Therapie der Frühgeburtlichkeit sind nicht ausreichend wirksam (Bettruhe, Hydratation, Sedierung), noch ungenügend erforscht (Gestagene) oder nur bei einer kleinen Untergruppe anwendbar (Entlastung bei Hydramnion, Notfallcerclage). Antibiotika sind nicht tokolytisch wirksam, sollten allerdings immer prophylaktisch bei PPROM, aber nicht bei blander vorzeitiger Wehentätigkeit ohne Infektionszeichen angewendet werden.

\section{Korrespondierender Autor \\ PD Dr. E. Beinder}

Department Frauenheilkunde, Universitätsspital Frauenklinikstraße 10, 8091 Zürich, Schweiz ernst.beinder@usz.ch

Interessenkonflikt. Es besteht kein Interessenkonflikt. Der korrespondierende Autor versichert, dass keine Verbindungen mit einer Firma, deren Produkt in dem Artikel genannt ist, oder einer Firma, die ein Konkurrenzprodukt vertreibt, bestehen. Die Präsentation des Themas ist unabhängig und die Darstellung der Inhalte produktneutral.

\section{Literatur}

1. Althuisius SM, Dekker GA, Hummel P, van Geijn HP (2003) Cervical incompetence prevention randomized cerclage trial: emergency cerclage with bed rest versus bed rest alone. Am J Obstet Gynecol 189: 907-911

2. Arlettaz R, Paraskevopoulos E, Bucher HU (2003) Triplets and quadruplets in Switzerland: comparison with singletons, and evolution over the last decade. J Perinat Med 31: 242-250
3. Blondel B, Kogan MD, Alexander GR et al. (2002) The impact of the increasing number of multiple births on the rates of preterm birth and low birthweight: in international study. Am J Public Health 92: 1323-1330

4. Carr SR, Aronson MP, Coustan DR (1990) Survival rates of monoamniotic twins do not decrease after 30 weeks of gestation. Am J Obstet Gynecol 163: 719-722

5. Cox SM, Sherman ML, Leveno KJ (1990) Randomized investigation of magnesium sulfate for prevention of preterm birth. Am J Obstet Gynecol 163: 767-772

6. Crowley P (2000) Prophylactic corticosteroids for preterm birth. Cochrane Database Syst Rev 2: CD000065

7. Crowther CA, Hiller JE, Doyle LW (2004) Magnesium sulphate for preventing preterm birth in threatened preterm labour (Cochrane Review). Cochrane Library, Iss 1, John Wiley, Chichester

8. Duckitt K, Thornton S (2002) Nitric oxide donors for the treatment of preterm labour. Cochrane Database Syst Rev 2002 CD002860

9. Gyetvai K, Hannah ME, Hodnett ED, Ohlsson A (1999) Tocolytics for preterm labor: a systematic review. Obstet Gynecol 94: 869-877

10. Holleboom CA, Merkus JM, van Elferen LW, Keirse MJ (1996) Double-blind evaluation of ritodrine sustained release for oral maintenance of tocolysis after active preterm labour. Br J Obstet Gynaecol 103: 702-705

11. Jeyabalan A, Caritis SN (2002) Pharmakologic inhibition of preterm labour. Clin Obstet Gynecol 45: 99-113

12. Keirse MJNC (1995) New perspectives for the effective treatment of preterm labour. Am J Obstet Gynecol 173: 618-628

13. Kenyon SL, Taylor DJ, Tarnow-Mordi W (2001) Broad spectrum antibiotics for preterm, prelabour rupture of fetal membranes: the ORACLE I randomised trial. ORACLE collaborative Group. Lancet 357: 979-988

14. Kenyon SL, Taylor DJ, Tarnow-Mordi W (2001) Broad spectrum antibiotics for spontaneous preterm labour: The ORACLE II randomised trial. Lancet 357: 989-994

15. King J, Flenady V (2000) Antibiotics for preterm labour with intact membranes. Cochrane Database Syst Rev 2: CD000246

16. King JF, Grant A, Keirse MJ, Chalmers I (1998) Beta-mimetics in preterm labor: an overview of randomized, controlled trials. Br J Obstet Gynaecol 95: 211-222

17. King JF, Flenady VJ, Papatsonis DNM, Dekker GA, Carbonne B (2003) Calcium channel blockers for inhibiting preterm labour (Cochrane Review). Cochrane Library, Iss 4, John Wiley, Chichester

18. King J, Flenady V, Cole S, Thornton S (2005) Cyclooxygenase (COX) inhibitors for treating preterm labour. Cochrane Database Syst Rev (2): CD001992

19. Lewis R, Mercer BM, Salama M, Walsh MA, Sibai BM (1996) Oral terbutaline after parenteral tocolysis: a randomized, double-blind, placebo controlled trial. Am J Obstet Gynecol 175: 834-837

20. Lydon-Rochelle M, Holt VR, Easterling TR, Martin DP et al. (2001) Risk of uterine rupture during labor among women with a prior cesarean delivery. N Engl J Med 345: 3-8

21. Martin JA, Hamilton BE, Ventura SJ et al. (2003) Births: final data for 2002. Natl Vital Stat Rep 52: 1113

22. Meis PJ, Aleman A (2004) Progesterone treatment to prevent preterm birth. Drugs 64: 2463-2474 
23. Moutquin JM, Sherman D, Cohen H et al. (2000) Double-blind, randomized, and controlled trial of atosiban and ritodrine in the treatment of preterm labor: a multicenter effectiveness and safety study. Am J Obstet Gynecol 182: 1191-1199

24. NIH Consensus Development Panel (1995) Effect of corticosteroids for fetal maturation on perinatal outcomes. JAMA 273: 413-418

25. Norton ME, Merill J, Cooper BA et al. (1993) Neonatal complications after the administration of indomethacin for preterm labor. N Engl J Med 329;1602-1607

26. Oei SG, Oei SK, Brölmann HAM (1999) Myocardial infarctation during nifedipine therapy for preterm labor. N Engl J Med 340: 154-155

27. Olatunbosun OA, al-Nuaim L, Turnell RW (1995) Emergency cerclage compared with bed rest for advanced cervical dilatation in pregnancy. Int Surg 80: 170

28. Pijnenborg JM, Oei SG (1999). The monoamniotic twin: a riskful event. Eur J Obstet Gynecol Reprod Biol 86: 51-53

29. Ricci JM, Hariharan S, Helfgott A et al. (1991) Oral tocolysis with magnesium chloride: a randomized controlled prospective clinical trial. Am J Obstet Gynecol 165: 603-610

30. Romero R, Sibai BM, Sanchez-Ramos L et al. (2000) An oxytocin receptor antagonist (atosiban) in the treatment of preterm labor: a randomized, double blind, placebo-controlled trial with tocolytic rescue. Am J Obstet Gynecol 182: 1191-1199

31. Sanchez-Ramos L, Kaunitz Am, Delke I (2005) Progestational agents to prevent preterm birth: a meta-analysis of randomized controlled trials. Obstet Gynecol 105: 273-279

32. Spätling L, Fallenstein F, Schneider H, Dancis J (1989) Bolus tocolysis: treatment of preterm labor with pulsatile administration of a beta-adrenergic agonist. Am J Obstet Gynecol 160: 713-717

33. The Worldwide Atosiban Versus Beta-agonists Study group (2001) Effectiveness and safety of the oxytocin antagonist atosiban versus beta-adrenergic agonists in the treatment of preterm labour. $\mathrm{Br}$ J Obstet Gynaecol 108: 133-142

34. Valenzuela GJ, Sanchez-Ramos L, Romero R et al. (2000) Maintenance treatment with the oxytocin antagonist atosiban. The atosiban PTL-098 study group. Am J Obstet Gynecol 182: 1184-1190

35. Van Veen AJ, Pelinck MJ, van Pampus MG, Erwich JJHM (2004) Severe hypotension and fetal death due to tocolysis with nifedipine. Br J Obstet Gynaecol 111: 1-2
Der Gynäkologe bietet jeden Monat umfassende und aktuelle Beiträge zu interessanten Themenschwerpunkten aus allen Bereichen der Gynäkologen und Geburtshilfe.

Möchten Sie ein bereits erschienenes Heft nachbestellen? Die folgenden Ausgaben können Sie direkt bei unserm Kundenservice zum Preis von EUR 27,- beziehen:

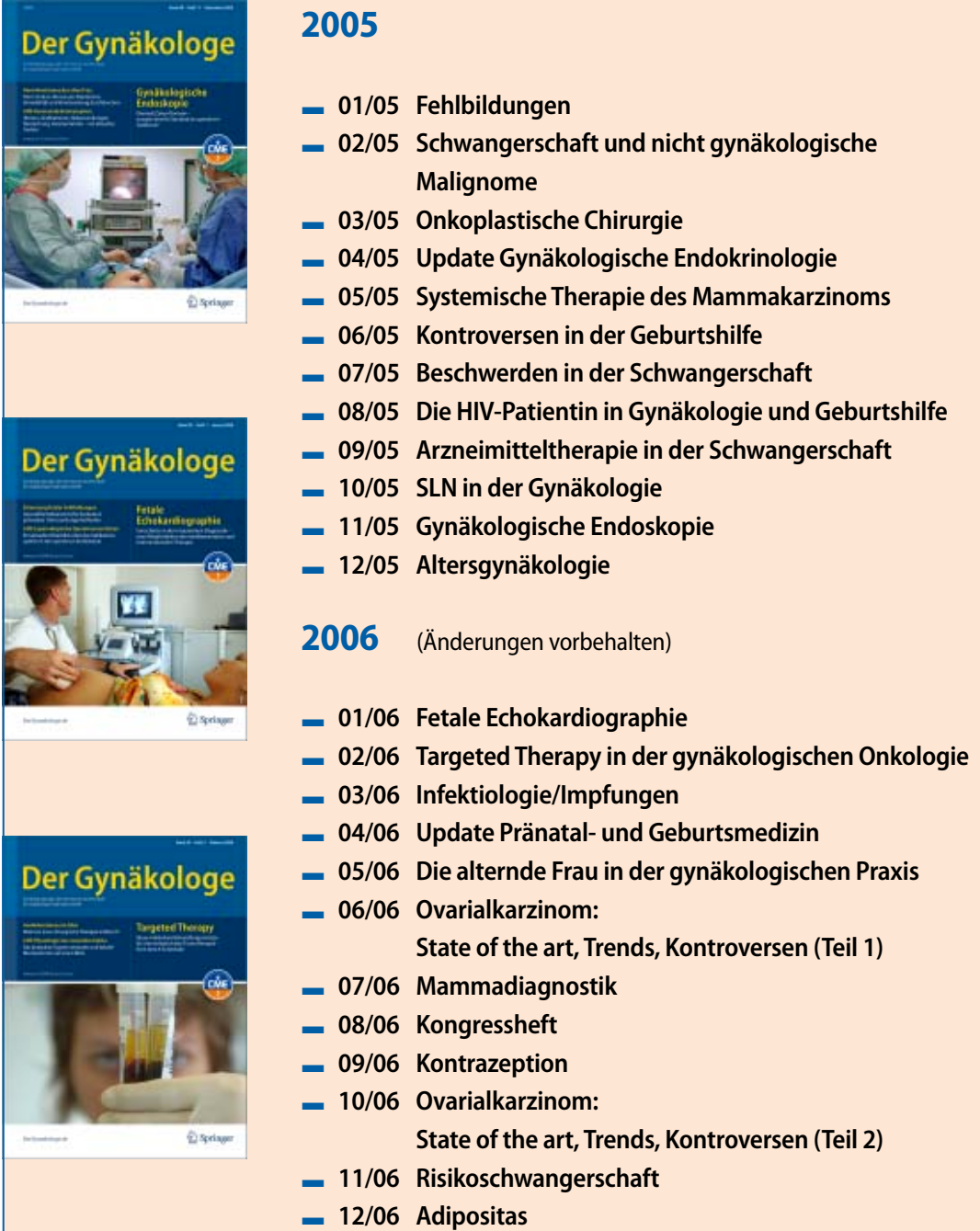

So erreichen Sei unseren Kundenservice:

Springer Medizin Verlag GmbH

Kundenservice Zeitschriften

Haberstraße 7

69126 Heidelberg

Tel. $+496221345-4303$

Fax: +496221 345-4229

E-Mail: SDC-journals@springer.com 